\title{
High Magnetic Field Transport and Photoluminescence in Doped InGaAs/InP Superlattices
}

\author{
A.B.Henriques, L.K.Hanamoto, R.F.Oliveira \\ Instituto de Física, Universidade de São Paulo, \\ Caixa Postal 66318, 05315-970 São Paulo, Brazil \\ P.L. Souza, L.C.D. Gonçalves, and B. Yavich \\ Centro de Estudos em Telecomunicaçôes, \\ Pontifícia Universidade Católica, \\ 22453-900 Rio de Janeiro, Brazil \\ Received February 6, 1999
}

\begin{abstract}
Lattice-matched $\operatorname{InP} / \operatorname{In}_{x} \mathrm{Ga}_{1-x}$ As short period superlattices $(x=0.53) \delta$-doped with $\mathrm{Si}$ in the middle of the In P barriers were studied. The samples had a high carrier concentration which filled two minibands. In addition to a peak associated with the electrons from the second miniband, E2, the Shubnikov-de Haas spectra showed a well resolved doublet structure that is assigned to $E 1$ electrons of superlattice wave vectors $k_{z}=0$ and $k_{z}=\pi / d$. From the lineshape of the Shubnikov-de Haas oscillations, an $E 1$ quantum mobility of $970 \mathrm{~cm}^{2} / \mathrm{Vs}$ was deduced, which represents an increase of about $40 \%$ over the value for periodically delta-doped semiconductors. The photoluminescence exhibits a band at photon energies higher than the InGaAs bandgap and whose FWHM approximates the Fermi energy of the confined carriers. Thus the photoluminescence observed is consistent with the recombination of electrons confined by the superlattice potential and photoexcited holes.
\end{abstract}

\section{Introduction}

In doped superlattices electrons are confined by a periodic potential in one dimension which splits the continuous conduction band into a set of discrete energy minibands. By changing the thickness of the component layers and the doping density, the energy spectrum of carriers can be tuned within a wide range. This characteristic makes doped superlattices a unique system in which to study the physical properties of an electronic system with a dimension between 2 and 3 , whereby such interesting effects as negative differential resistance [1] and chaotic transport $[2,3]$ can be observed.

In addition to the lineshape of the periodic potential and the density of confined carriers, the lifetime of the single-particle states is also a key factor in determining the electronic properties of the superlattice. Our previous work on periodically delta-doped superlattices $[4,5]$ has shown that the lifetime of the electronic state at the Fermi energy increases with the average distance between the electron spatial distribution and the ionised impurity sheet. In this work we attempted to reduce the rate of scattering by increasing the distance between electrons and impurities. Results obtained on doped InP/InGaAs superlattices are presented. The doping sheet of $\mathrm{Si}$ atoms was placed in the InP barriers. In such a structure electrons are repelled by the InP barriers, and this repulsion favours their spatial separation from the impurity atoms, which leads to longer single-particle lifetimes.

\section{Experimental}

The superlattices were grown at $640^{\circ} \mathrm{C}$ by LP-MOVPE in an AIX200 reactor at 20mbar. On a (100) Fe-doped InP substrate, first a $800 \AA$ thick undoped buffer layer was grown, followed by a 15 period multiple quantum well structure composed of a $50 \AA$ thick lattice matched InGaAs quantum well and a $50 \AA$ thick InP barrier. Finally, a $300 \AA \mathrm{InP}$ cap layer was deposited. A Si delta layer with a sheet carrier concentration equal to $4.5 \times 10^{13} \mathrm{~cm}^{-2}$ was introduced in the middle of the InP barriers. Silane was used as the Si source and was introduced in the growth chamber 5s after interruption of the InP growth. The doping plane was deposited during 
15 seconds after which the silane flux was halted. The growth of the second half of the barriers was triggered 5 seconds later. The period of the MQW structure was determined by x-ray diffractometry. The Shubnikovde Haas spectrum of the sample was measured at $4.2 \mathrm{~K}$ in magnetic fields of intensity 0-16 T. PL spectra were measured at $4.2 \mathrm{~K}$ using a set-up whereby light was conveyed to and from the sample using optical fibres.

\section{Results}

Figure 1(a) shows the Shubnikov-de Haas (SdH) spectrum of sample 326, and Figure 1(b) shows its Fourier transform. The doublet structure seen in 1(b) at
$\mathrm{B} \sim 80 \mathrm{~T}$ is due to a resolution of the belly $\left(k_{z}=0\right)$ and neck $\left(k_{z}=\pi / d\right)$ extremal cross sections of the Fermi surface associated with the lowest energy miniband $E 1$, whereas the peak at $\mathrm{B} \sim 40 \mathrm{~T}$ is due to the pocket of electrons confined in miniband $E 2$. Based on the oscillation frequencies observed, the electronic energy spectrum was calculated following the same scheme as used previously for periodically delta-doped structures [4]. In the present structures, however, an additional complication arises from the difference in the effective mass of electrons in bulk InGaAs and InP. The miniband energy spectrum was calculated by solving the Schrödinger equation, analogously to the Ben Daniel-Duke procedure for quantum wells [6]

$$
\left[-\frac{\hbar^{2}}{2 m(z)} \frac{d}{d z} \frac{1}{m(z)} \frac{d}{d z}+V(z)\right] \chi_{n}\left(k_{z}, z\right)=E_{n}\left(k_{z}\right) \chi_{n}\left(k_{z}, z\right)
$$

and the Fermi surface was obtained from the equation

$$
\frac{\hbar^{2} k_{\perp}^{2}}{2 m_{\perp}\left(n, k_{z}\right)}+E_{n}\left(k_{z}\right)=E_{F}
$$

where $m_{\perp}\left(n, k_{z}\right)$ is the effective mass associated with the movement of electrons in a direction perpendicular to the superlattice axis,

$$
\frac{1}{m_{\perp}\left(n, k_{z}\right)}=\int_{-d / 2}^{d / 2} \frac{\left|\chi_{n}\left(k_{z}, z\right)\right|^{2}}{m(z)} d z
$$

where $d$ is the superlattice period, $m(z)$ is the electronic effective mass in the bulk of the semiconductor material at the position $z$ along the growth axis, and $\chi_{n}\left(k_{z}, z\right)$ is the electronic wave function. The confining potential, $\mathrm{V}(\mathrm{z})$, was approximated either by the Kronig-Penney (KP) form, assuming an InP/InGaAs conduction band offset of $230 \mathrm{meV}$, or obtained from a full self-consistent (SC) solution of equation (1) and the Poisson equation, including the exchange and correlation correction in the local density approximation. In the latter case the ionised $\mathrm{Si}$ atoms were assumed to be obey a gaussian distribution with a full width at halfheight of $\delta=0.05 a_{B}$ ( $7 \AA$ for InGaAs) or $\delta=0.21 a_{B}$ ( $30 \AA$ for InGaAs). A comparison of the results of these calculations is shown in Figure 2. This figure shows that the energy separation between the Fermi level and the bottom of miniband $E 1,\left(\phi_{E 1}\right)$ obtained from the KP and the SC calculations agree within $10 \%$ if the density of carriers is less than $n_{S}=10 a_{B}^{-2}\left(5.2 \times 10^{12} \mathrm{~cm}^{-2}\right.$ per superlattice period. However, the same does not hold in the case of excited minibands. For instance, for $n_{S}=8 a_{B}^{-2}\left(4.1 \times 10^{12} \mathrm{~cm}^{-2}\right.$ for InGaAs $)$ the SC calculations with $\delta=0.05 a_{B}$ gives $\phi_{E 2}$ which is more than 2 times greater than the value obtained from the KP model; this ratio falls to 1.8 if $\delta=0.21 a_{B}$ is assumed. Notice that the disagreement between the KP and the $\mathrm{SC}$ models is reduced for a larger $\delta$, which is because the KP potential approximates better the Hartree SC potential for a homogeneous distribution of doping atoms.

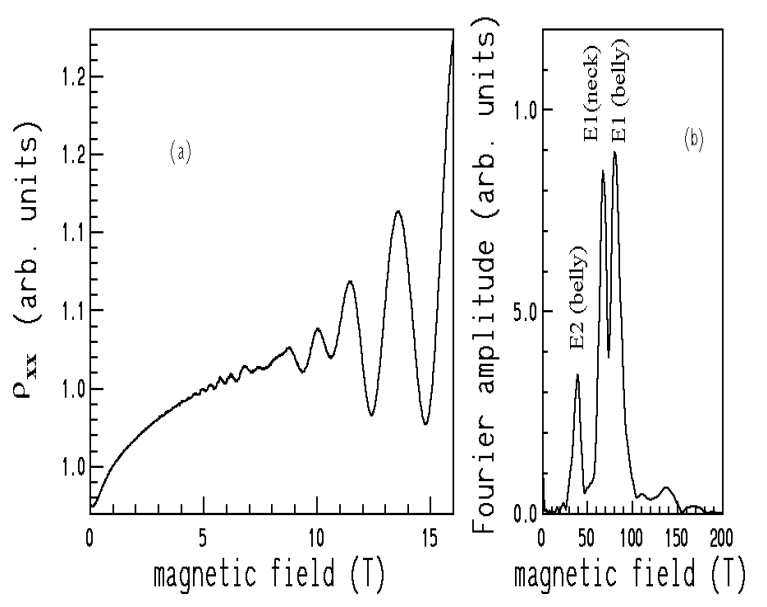

Figure 1. Shubnikov-de Haas oscillations (a) and its Fourier transform (b) measured at $4.2 \mathrm{~K}$ for sample No.326. 
Table I shows a comparison between the experimental SdH frequencies and the theoretical ones deduced from the KP and SC approximations, assuming $n_{S}=3.9 \times 10^{12} \mathrm{~cm}^{-2}$. This value of $n_{S}$ was chosen because it gives theoretical $\mathrm{SdH}$ frequencies which approximate best the experimental values for miniband $E 1$ - for $\delta=0.05 a_{B}$, the difference between theory and experiment is less than $2 \%$. However, a discrepancy remains in respect to the oscillations associated with $E 2$ electrons, which worsens if $\delta$ increases. This discrepancy is probably due to the effects of residual background doping, non-abruptness of the interfaces and fluctuations in the alloy composition, characteristics which were not taken into account in the theory. Our reasoning is based on the observation that the $E 2$ miniband parameters are much more sensitive to the details of the structure than the $E 1$ ones, as Figure 2 demonstrates.

Table I. Experimental and theoretical SdH frequencies for sample No.326. The theoretical values were obtained assuming $d=100 \AA$ and $n_{S}=3.9 \times 10^{12} \mathrm{~cm}^{-2}$.

\begin{tabular}{c|c|c|c|c|c|c|c|c}
\hline \hline \multicolumn{3}{c|}{ Belly $(E 1)(\mathrm{T})$} & \multicolumn{3}{c|}{ Neck $(E 1)(\mathrm{T})$} & \multicolumn{3}{c}{ Belly (E2) (T) } \\
\hline Exper. & SC $(\delta=7 \AA)$ & KP & Exper. & SC $(\delta=7 \AA)$ & KP & Exper. & SC $(\delta=7 \AA)$ & KP \\
\hline 79.1 & 80.1 & 83.7 & 69.1 & 68.3 & 69.6 & 38.7 & 21.9 & 7.2 \\
\hline \hline
\end{tabular}

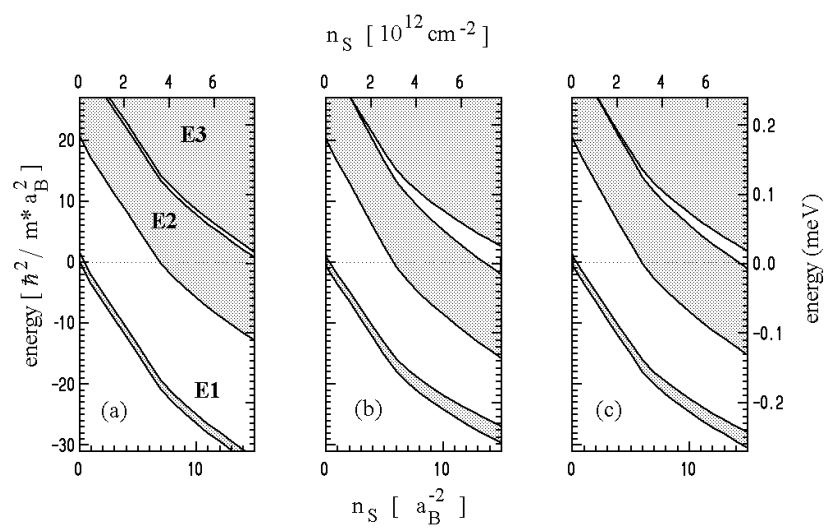

Figure 2. Energy miniband structure for a InGaAs/InP superlattice with period $d=100 \AA$ ( $\mathrm{InP}$ and InGaAs layers of equal width). The Fermi level defines the zero of energy. (a) Kronig-Penney model (b) Self-consistent model with $\delta=7 \AA$ (c) Self consistent model with $\delta=30 \AA$.

Assuming $n_{S}=3.9 \times 10^{12} \mathrm{~cm}^{-2}$ and $\delta=7 \AA$ the effective mass $m_{\perp}\left(E 1, k_{z}\right)$ was deduced from Eq. (3), and it changed from $0.051 m_{0}$ at $k_{z}=0$ to $0.049 m_{0}$ at $k_{z}=\pi / d$. The nearly constant value of $m_{\perp}$ allows us to assume that the $E 1 \mathrm{SdH}$ oscillations will be well described by the tight-binding model [7],

$$
\frac{\Delta \rho_{x x}}{\rho_{0}} \sim-2 \frac{X}{\sinh X} \exp ^{-\alpha u} J_{0}(\beta u) \cos (\omega u)
$$

where $X=2 \pi^{2} k_{B} T / \hbar \omega_{c}, \omega_{c}$ is the in-plane cyclotron frequency, $\alpha=\pi / \mu_{Q}, \mu_{Q}$ is the quantum mobility in the $E 1$ miniband, $\beta=\pi m_{\perp} \Delta / \hbar e, \Delta$ is the width of the miniband, $\omega=2 \pi m_{\perp}\left(\phi_{E 1}-\Delta / 2\right) / \hbar e$, and $J_{0}$ is the Bessel function of zero order. We have isolated the $E 1$ oscillations from the rest of the $\mathrm{SdH}$ spectrum using Fourier techniques. The $\mathrm{SdH}$ oscillations so obtained were fitted using equation (4) to output the quantum mobility $\mu_{Q}$. The values of the $E 1$ parameters $\Delta$ and $\phi_{E 1}$ were allowed to evolve from the initial values output by the $\mathrm{SC}$ calculation. As shown in Fig.3, the theoretical curve is in excellent agreement with the experimental one if the quantum mobility is set at $\mu_{Q}=970 \mathrm{~cm}^{2} / \mathrm{Vs}$. The quantum mobility for a delta-doping of the same period $\left(0.72 a_{B}\right)$ and same carrier density $\left(7.5 a_{B}^{-2}\right)$ is less than $0.25 e a_{B}^{2} / \hbar$ [8], i.e. $700 \mathrm{~cm}^{2} / \mathrm{Vs}$ for InGaAs. Thus, by separating the electrons and impurities, an improvement of nearly $40 \%$ in quantum mobility was achieved.

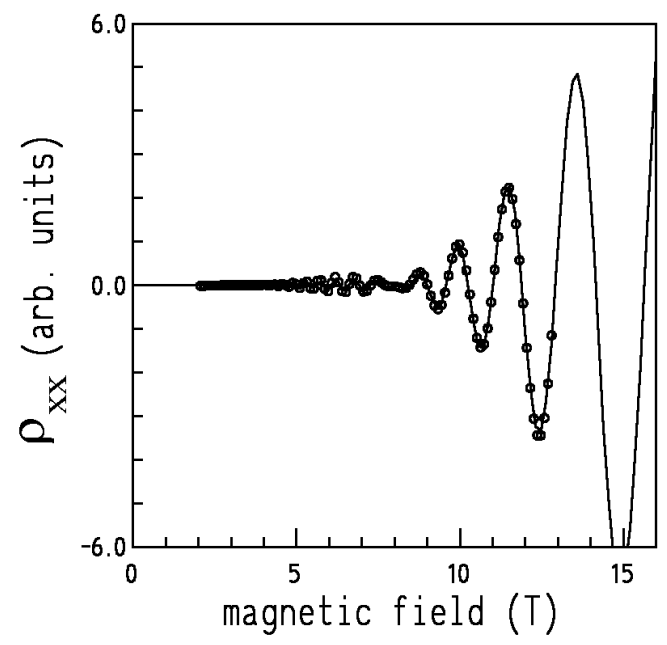

Figure 3. Isolated SdH oscillations due to electrons in miniband E1 and theoretical result by use of Eq.(4).

The photoluminescence of the superlattice was measured at $4.2 \mathrm{~K}$ and it is shown in Figure 4 . Notice that the luminescence band occurs at an energy well above 
the InGaAs bandgap of $0.81 \mathrm{eV}$, and the width of the luminescence band is about $150 \mathrm{meV}$, which approximates quite well the value of the E1 Fermi energy, $\phi_{E 1} \sim 190 \mathrm{meV}$, as deduced from the SC calculations. This is supportive of associating the observed luminescence with a recombination of photoexcited holes and the Fermi sea of electrons confined by the superlattice potential.

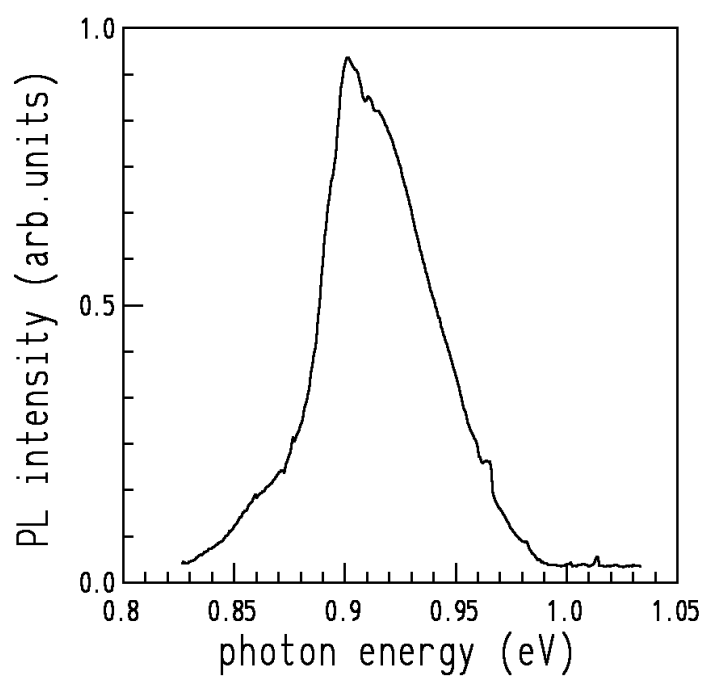

Figure 4. Photoluminescence of the InGaAs superlattice at $4.2 \mathrm{~K}$.

\section{Summary}

Doped InP/InGaAs superlattices were studied. Modelling of the structures showed that the Kronig-Penney approximation describes with acceptable accuracy the ground-state electronic miniband, but not the excited ones. Shubnikov-de Haas spectra of the samples showed a very well resolved doublet structure associated with the belly and neck orbits in the E1 miniband. The quantum mobility for electrons confined in the $E 1$ miniband was deduced to be $970 \mathrm{~cm}^{2} / \mathrm{Vs}$. This represents an improvement of nearly $40 \%$ in comparison with periodically delta-doped structures. The photoluminescence spectrum showed an emission band which is consistent with the recombination of photoexcited holes and electrons confined by the superlattice potential.

\section{Acknowledgements}

This work was supported by FAPESP, CNPq, and CAPES - Brazilian funding agencies.

\section{References}

[1] E. Schomburg et al, Phys. Rev. B 58, 4035 (1998).

[2] K.J. Luo, H.T. Grahn, K.H. Ploog and L.L. Bonilla, Phys. Rev. Lett. 81, 1290 (1998).

[3] F.W. Sheard, Proceedings of the the 24th International Conference on the Physics of Semiconductors (to be published).

[4] A.B. Henriques, L.C.D. Gonçalves, N.F. Oliveira, Jr., P.L. Souza \& B. Yavich, Phys. Rev. B 55, 13072 (1997).

[5] A.B. Henriques, L.C.D. Gonçalves, N.F. Oliveira, Jr., S.M. Shibli, P.L. Souza \& B. Yavich, Zeitschrift für Physik B 104, 457 (1997).

[6] G.Bastard, Wave mechanics applied to semiconductor heterostructures (Les Editions de Physique, Les Ulis, 1988), p.73.

[7] A.B. Henriques, Phys. Rev. B 50, 8658 (1994).

[8] A.B. Henriques, Phys. Rev. B 53, 16365 (1996). 